\title{
Development of a proteoliposome model to probe transmembrane electron-transfer reactions
}

\author{
Gaye F. White", Zhi Shi†, Liang Shi†, Alice C. Dohnalkova†, James K. Fredrickson†, John M. Zachara†, Julea N. Butt*, \\ David J. Richardson* and Thomas A. Clarke*1 \\ "Centre for Molecular and Structural Biochemistry, School of Biological Sciences and School of Chemistry, University of East Anglia, Norwich NR4 7TJ, U.K., and \\ †Pacific Northwest National Laboratory, P.O. Box 999, 902 Battelle Boulevard, Richland, WA 99352, U.S.A.
}

\begin{abstract}
The mineral-respiring bacterium Shewanella oneidensis uses a protein complex, MtrCAB, composed of two decahaem cytochromes brought together inside a transmembrane porin to transport electrons across the outer membrane to a variety of mineral-based electron acceptors. A proteoliposome system has been developed that contains Methyl Viologen as an internalized electron carrier and valinomycin as a membraneassociated cation exchanger. These proteoliposomes can be used as a model system to investigate MtrCAB function.
\end{abstract}

\section{MtrCAB is an outer membrane electron transporter}

Bacterial mineral respiration requires electrons to be transported to the cell exterior in order to reduce extracellular insoluble electron acceptors. The proteins essential for these processes have been identified and studied in model mineralrespiring organisms such as Shewanella oneidensis [1,2]. These studies indicate that multihaem proteins co-operate to conduct electrons from the cell interior across otherwise impermeable cell membranes, allowing them to react with external electron acceptors such as $\mathrm{Fe}(\mathrm{III})$ minerals [3-5]. The MtrCAB complex is responsible for electron transfer across the outer membrane of $S$. oneidensis. This complex contains the periplasmic decahaem MtrA and membraneassociated decahaem MtrC that is exposed on the cell surface. These two cytochromes meet and exchange electrons inside a transmembrane sheath, MtrB [6]. Structural studies of the $\mathrm{MtrC}$ homologue MtrF show that the ten haems are arranged in a staggered cross which would allow for quadridirectional electron transport with one membrane-facing entry and three exit sites on the protein surface [7]. However, there is much debate over how such systems deliver charge to external redox partners with a number of potential electron-transfer mechanisms being proposed [7].

Reconstitution of transport proteins into phospholipid bilayers is a recognized way of studying biochemical and biophysical parameters in a model membrane environment $[8,9]$. In a previous study of $\mathrm{MtrCAB}$, proteoliposomes incorporating the redox dye MV (Methyl Viologen) were reduced with dithionite [6]. The interior could only be reduced if the protein complex were present, demonstrating that MtrCAB has the ability to move electrons across an otherwise impermeable lipid bilayer. Since these earlier studies, this

Key words: microbe-mineral electron transfer, MtrCAB, multihaem cytochrome, proteoliposome, Shewanella oneidensis.

Abbreviations used: MV, Methyl Viologen; SHE, standard hydrogen electrode.

${ }^{1}$ To whom correspondence should be addressed (email tom.clarke@uea.ac.uk). methodology has been tested and optimized with a view to wider applications that can facilitate detailed kinetic and mechanistic studies of interactions that involve electron transport through MtrCAB and, ultimately, be used to study other protein systems connected with transmembrane electron-transfer processes.

\section{Efficient electron transfer through MtrCAB in proteoliposomes}

Proteoliposomes offer the opportunity to model the electrontransfer pathway that occurs in a bacterial cell, where the encapsulated interior has a negative potential compared with the exterior environment and the only exit pathway for electrons is through the electron transporting protein complex. Thus, when a suitable electron acceptor is introduced into the exterior environment, electrons will flow out through the protein complex as they would in vivo. The $\mathrm{MV}$ inside the MtrCAB proteoliposomes is designed to act both as a means of storing charge in its reduced state that will readily be released to external electron acceptors and also as an integral redox indicator to follow electron-transfer reactions. The redox potential for the one-electron reduction of MV is $\mathrm{MV}^{2+} / \mathrm{MV}^{\bullet+}=-0.45 \mathrm{~V}$ compared with the SHE (standard hydrogen electrode) at $\mathrm{pH} 7.0$ and the colour changes from colourless to blue $[10,11]$. The redox potentials of $\mathrm{Fe}(\mathrm{III})$ oxides are typically between $-0.3 \mathrm{~V}$ and $+0.06 \mathrm{~V}$ compared with the SHE at $\mathrm{pH} 7.0[12,13]$. If there is a suitable pathway through the lipid bilayer, electrons should readily transfer from the encapsulated reduced MV to externally located electron acceptors such as soluble $\mathrm{Fe}(\mathrm{III})$ chelates or insoluble $\mathrm{Fe}(\mathrm{III})$ minerals.

However, to ensure that electron transfer occurs solely via the protein complex, it is imperative to ensure that the lipid bilayer remains impermeable to charged solutes for the duration of the experiment. The reduced proteoliposomes were centrifuged to sediment them and the supernatant 
Figure 1 | Improving the stability of proteoliposomes for electron-transfer investigations

(A) Schematic representation of the MtrCAB proteoliposomes. The MtrCAB complex is shown mediating electron transfer between oxidized and reduced forms of $M V\left(M V^{2+} / M V^{\bullet+}\right)$ and external electron acceptors or donors $\left(X^{+} / X\right)$. Valinomycin (V) functions as a $\mathrm{K}^{+}$-specific transporter. (B) MtrCAB proteoliposomes prepared with and without valinomycin were incubated with $100 \mu \mathrm{M}$ sodium dithionite for $20 \mathrm{~min}$, then centrifuged for $1 \mathrm{~h}$ at $280000 \boldsymbol{g}$ to sediment the proteoliposomes. Any MV released remained in the supernatant. From the absorbance profile, addition of $100 \mu \mathrm{M}$ sodium dithionite to the supernatant from the valinomycin-free MtrCAB proteoliposomes showed that $10 \mu \mathrm{M} \mathrm{MV}$ had been released (broken line). Absorbance after addition of $100 \mu \mathrm{M}$ sodium dithionite to the supernatant from MtrCAB proteoliposomes containing $10 \mathrm{nM}$ valinomycin showed that less than $1 \mu \mathrm{M}$ MV was in the supernatant (continuous line).
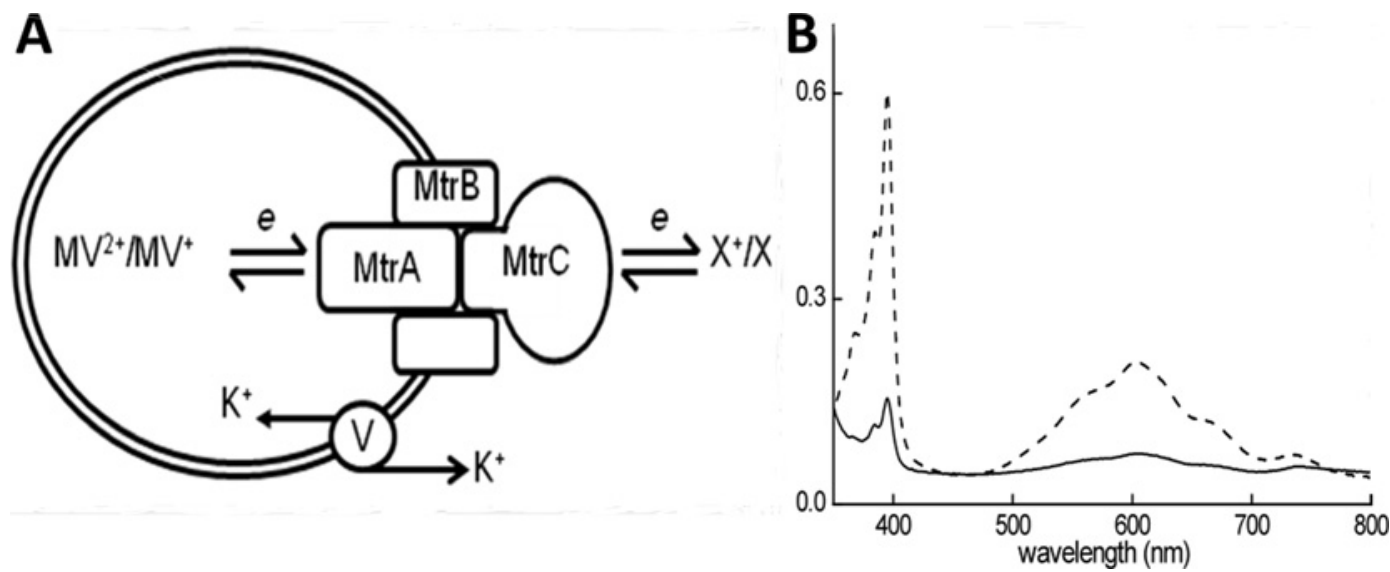

was analysed to determine whether any MV had been released. After 20 min of anaerobic incubation with dithionite followed by $1 \mathrm{~h}$ of centrifugation, approximately $30 \%$ of the MV was found in the supernatant. Thus the encapsulation of the MV was not maintained after the electron transfer, as may be expected when bulk charge imbalances build up across the lipid bilayer and different redox states of MV, including $\mathrm{MV}^{0}$, may be formed [14]. Valinomycin is known to form channels that selectively transport $\mathrm{K}^{+}$ions through lipid bilayers [15]. $\mathrm{KCl}$ and valinomycin were added to the system so that charge differentials caused by electron transport could be balanced by the migration of positively charged ions. This scheme (Figure 1A) led to greatly enhanced retention of MV inside the proteoliposomes with less than $3 \%$ leakage into the surrounding medium (Figure 1B).

\section{Biophysical characterization of MtrCAB proteoliposomes}

Proteoliposomes were prepared by detergent-mediated reconstitution of Triton X-100-solubilized MtrCAB into blank phosphatidylcholine liposomes. Freeze-dried phosphatidylcholine was resuspended in MV solution and subjected to extrusion, sonication and freeze-thaw cycles to produce separate regularly sized spheroids formed from unilamellar lipid bilayers impermeable to charged species. Various amounts of protein solution were added to aliquots of blank liposome suspension to give 0-50 nM MtrCAB. These were incubated with Biobeads for $1 \mathrm{~h}$ to remove detergent. Repeated wash steps were performed using centrifugation at $280000 \mathrm{~g}$ to remove MV external to blank liposomes and proteoliposomes.

The physical attributes of the proteoliposomes were characterized using dynamic light scattering and transmission electron microscopy, showing they were separate regularly sized spheroids with an average diameter of approximately $500 \mathrm{~nm}$ (Figure 2). After purging with $\mathrm{O}_{2}$-free $\mathrm{N}_{2}$ and maintaining anaerobicity, a 10 -fold dilution of blank liposomes was treated with sodium dithionite. The characteristic absorbance spectrum of reduced $\mathrm{MV}, \mathrm{MV}^{\bullet+}$, was not observed until $1 \%$ Triton X-100 was added to disrupt the lipid bilayer and release the contents of the liposomes (Figure 3A). This shows that the liposomal membrane is impermeable to electron transfer and there was no diffusion of MV or sodium dithionite across the lipid bilayer during the time course of the experiment. When MtrCAB proteoliposomes were subjected to the same treatment, the absorbance spectrum of reduced MV was observed after sodium dithionite was added, showing that electrons were able to cross to the interior of the liposomes solely by conduction through the MtrCAB complex (Figure 3B). The absorbance at $600 \mathrm{~nm}$ increased and reached a steady state after approximately $10 \mathrm{~min}$.

The MV content of the proteoliposome suspensions was determined using $\mathrm{MV}^{\bullet+} \varepsilon_{606}=13700 \mathrm{M}^{-1} \cdot \mathrm{cm}^{-1}[10]$. The efficiency of MV encapsulation and reduction was inversely proportional to the amount of protein incorporated, with optimal efficiency at $1 \mathrm{nM}$ MtrCAB. Estimates of the size and volume encompassed by the optimized proteoliposomes were used to estimate their number in relation to the amount of protein added. Protein incorporation was estimated by 
Figure 2 | Physical characterization of proteoliposomes

(A) Transmission electron microscopy image of an MtrCAB proteoliposome. The inset shows the lipid bilayer of a prepared liposome. (B) Size distribution (by diameter in $\mathrm{nm}$ ) of particles in a suspension of MtrCAB proteoliposomes measured by dynamic light scattering.
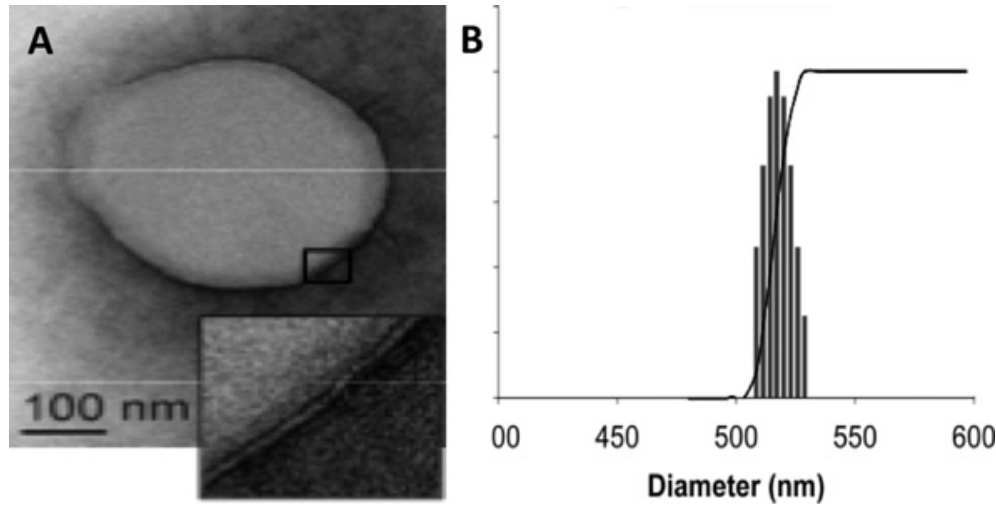

Figure 3 Reduction of MV encapsulated in liposomes and proteoliposomes

(A) Absorbance profiles of liposomes containing no protein (continuous grey line), 10 min after addition of $100 \mu \mathrm{M}$ sodium dithionite (continuous black line), followed by addition of 1\% Triton X-100 (broken line). Absorbance with maxima at 400 and $600 \mathrm{nM}$ corresponds to reduced MV. Reduction of MV was only observed after the liposome contents had been released by detergent. (B) Absorbance profiles of proteoliposomes prepared with $1 \mathrm{nM}$ MtrCAB (continuous grey line) $10 \mathrm{~min}$ after addition of $100 \mu \mathrm{M}$ sodium dithionite (continuous black line) followed by 1\% Triton X-100 (broken line). Reduction of MV (27 $\mu \mathrm{M}$ ) trapped within the proteoliposomes was observed on addition of sodium dithionite. After the trapped contents were released by the addition of 1\% Triton X-100, further reduction was observed ( $32 \mu \mathrm{M}$ reduced MV in total).

A

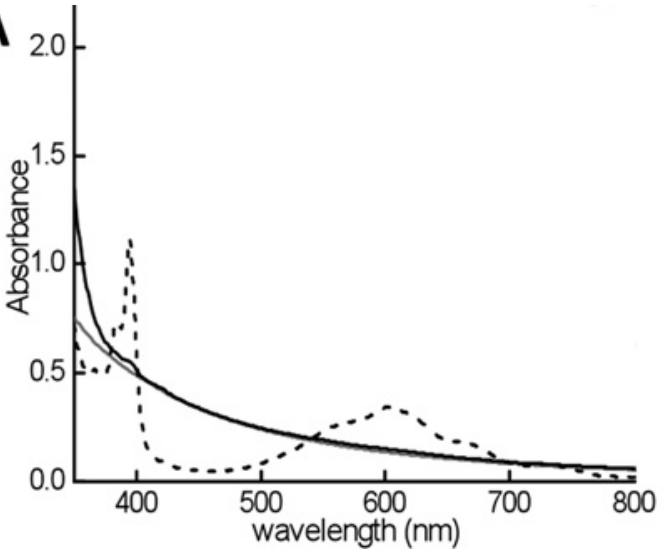

B

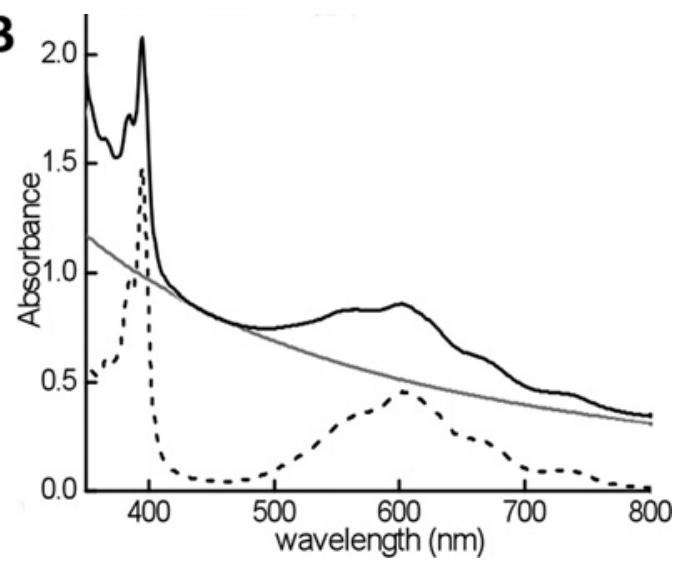

Western blots with MtrC-specific antibodies. It was found the most efficient reduction of the proteoliposome interior was observed when the molar ratio of protein to liposomes was approximately 10-200 MtrCAB molecules per liposome. In the optimized experiments, a total of $32 \mu \mathrm{M}$ MV was released from the proteoliposomes by detergent, of which approximately $80 \%$ could be reduced directly by dithionite via MtrCAB. In the earlier published work, additions of $0.2 \mu \mathrm{M}$ MtrCAB were used [6]. In this case, less than $1 \mu \mathrm{M}$ $\mathrm{MV}$ was trapped inside the proteoliposomes, of which less than $50 \%$ was reduced directly by dithionite via MtrCAB [6]. By incorporating a lower ratio of MtrCAB than used in the previous work and using $\mathrm{K}^{+}$and valinomycin to balance charge differentials, the proteoliposome methodology has been greatly improved.

\section{Applications for proteoliposome methodology}

The viability of the scheme shown in Figure 1(A) has been tested with MtrCAB as the electron transporter, MV on the interior and sodium dithionite on the exterior of the proteoliposomes. Under strictly anaerobic conditions, this produces stable proteoliposomes that encapsulate an interior with a negative potential that, apart from the MtrCAB electron-transport pathway, is isolated from the exterior 
environment. Ultimately, the aim is to introduce an electron acceptor into the medium surrounding the reduced proteoliposomes and spectroscopically follow electron transfer from inside to outside as $\mathrm{MV}^{\bullet+}$ reoxidizes to $\mathrm{MV}^{2+}$. In the first instance, it will be important to ensure that there are no other redox-active chemicals or mediators present. Thus anaerobicity must be maintained and there must be no excess dithionite remaining in the medium surrounding the proteoliposomes. Once the system can be established under these conditions, the external electron acceptor can be varied to investigate the kinetics of MtrCAB-mediated electron transfer to a range of soluble and insoluble electron acceptors. Indeed, many other factors can be varied, such as having interior redox partners with different potentials, use of different methods to reduce the interior, addition of mediators to the external environment, and incorporation of different quantities and types of proteins within the system. This methodology promises to be generally applicable to investigations of electron transfer processes in S. oneidensis and other organisms that partake in extracellular electron transfer.

\section{Funding}

This work was supported by the Biotechnology and Biological Sciences Research Council [grant number H007288/1] and the Subsurface Biogeochemical Research program (SBR)/Office of Biological and Environmental Research (BER), U.S. Department of Energy (DOE), and is a contribution of the Pacific Northwest National Laboratory (PNNL) Scientific Focus Area.

\section{References}

1 Shi, L., Richardson, D.J., Wang, Z.M., Kerisit, S.N., Rosso, K.M., Zachara, J.M. and Fredrickson, J.K. (2009) The roles of outer membrane cytochromes of Shewanella and Geobocter in extracellular electron transfer. Environ. Microbiol. Rep. 1, 220-227

2 Shi, L., Rosso, K.M., Clarke, T.A., Richardson, D.J., Zachara, J.M. and Fredrickson, J.K. (2012) Molecular underpinnings of Fe(III) oxide reduction by Shewanello oneidensis MR-1. Front. Microbiol. 3, 50
3 Ross, D.E., Ruebush, S.S., Brantley, S.L., Hartshorne, R.S., Clarke, T.A., Richardson, D.J. and Tien, M. (2007) Characterization of protein-protein interactions involved in iron reduction by Shewonello oneidensis MR-1. Appl. Environ. Microbiol. 73, 5797-5808

4 Wang, Z., Liu, C., Wang, X., Marshall, M.J., Zachara, J.M., Rosso, K.M. Dupuis, M., Fredrickson, J.K., Heald, S. and Shi, L. (2008) Kinetics of reduction of $\mathrm{Fe}$ (III) complexes by outer membrane cytochromes MtrC and OmcA of Shewanello oneidensis MR-1. Appl. Environ. Microbiol. 74, 6746-6755

5 Ross, D.E., Brantley, S.L. and Tien, M. (2009) Kinetic characterization of OmcA and MtrC, terminal reductases involved in respiratory electron transfer for dissimilatory iron reduction in Shewonello oneidensis MR-1. Appl. Environ. Microbiol. 75, 5218-5226

6 Hartshorne, R.S., Reardon, C.L., Ross, D., Nuester, J., Clarke, T.A., Gates, A.J., Mills, P.C., Fredrickson, J.K., Zachara, J.M., Shi, L. et al. (2009) Characterization of an electron conduit between bacteria and the extracellular environment. Proc. Natl. Acad. Sci. U.S.A. 106 22169-22174

7 Clarke, T.A., Edwards, M.J., Gates, A.J., Hall, A., White, G.F., Bradley, J., Reardon, C.L., Shi, L., Beliaev, A.S., Marshall, M.J. et al. (2011) Structure of a bacterial cell surface decaheme electron conduit. Proc. Natl. Acad. Sci. U.S.A. 108, 9384-9389

8 Banerjee, R.K. and Datta, A.G. (1983) Proteoliposome as the model for the study of membrane-bound enzymes and transport proteins. Mol. Cell. Biochem. 50, 3-15

9 Rigaud, J.-L., Pitard, B. and Levy, D. (1995) Reconstitution of membrane proteins into liposomes: application to energy-transducing membrane proteins. Biochim. Biophys. Acta 1231, 223-246

10 Watanabe, T. and Honda, K. (1982) Measurement of the extinction coefficient of the methyl viologen cation radical and the efficiency of its formatlon by semiconductor photocatalysis. J. Phys. Chem. $\mathbf{8 6}$ 2617-2619

11 Bockman, T.M. and Kochi, J.K. (1990) Isolation and oxidation-reduction of methylviologen cation radicals: novel disproportionation in charge-transfer salts by X-ray crystallography. J. Org. Chem. 55 4127-4135

12 Cornell, R.M. and Schwertmann, U. (2003) The Iron Oxides: Structure, Properties, Reactions, Occurrences and Uses, Wiley, Weinheim

13 Weber, K.A., Achenbach, L.A. and Coates, J.D. (2006) Microorganisms pumping iron: anaerobic microbial iron oxidation and reduction. Nat. Rev. Microbiol. 4, 752-764

14 Patterson, B.C., Thompson, D.H. and Hurst, J.K. (1988) Methyl viologen mediated oxidation-reduction across dihexadecylphosphate vesicles involves transmembrane diffusion. J. Am. Chem. Soc. 110, 3656-3657

15 Nichols, J.W. and Deamer, D.N. (1980) Net proton-hydroxyl permeability of large unilamellar liposomes measured by an acid-base titration technique. Proc. Natl. Acad. Sci. U.S.A. 77, 2038-2042

Received 1 May 2012

doi:10.1042/BST20120116 